“(C) 2018 IEEE. Personal use of this material is permitted. Permission from IEEE must be obtained for all other uses, in any current or future media, including reprinting/republishing this material for advertising or promotional purposes, creating new collective works, for resale or redistribution to servers or lists, or reuse of any copyrighted component of this work in other works." 


\title{
A Double-Voltage-Controlled Effective Thermal Conductivity Model of Graphene for Thermoelectric Cooling
}

\author{
Ning Wang, Hong-Wen Li, Can Ding, Li-Yun Shi, Hong-Zhi Jia, Zhong-Dao Ren, and Zi-Yi Zhao
}

\begin{abstract}
Graphene provides a new opportunity for thermoelectric study based on its unique heat transfer behavior controlled by the gate voltage. In this paper, an effective thermal conductivity model of graphene for thermoelectric cooling is proposed. The model is based on a double-voltage-controll mechanism. According to the law of Fourier heat conduction, an effective thermal conductivity model of thermoelectric cooling elements is derived with tunable external applied voltage. Combining with the voltage modulation characteristic for thermoelectric parameters, the regulation law of overall module thermal conductivity model is obtained with double-voltage-controlled feature. This model can be synchronously regulated by the gate voltage and external applied voltage. To verify correctness and effectiveness of the proposed model, a circuit simulation model using HSPICE tool is built based on the thermoelectric duality. The results show that the relative error between the proposed effective thermal conductivity and circuit simulation model is only $4 \%$ with the separately controlled external applied voltage at the maximum temperature difference. While the relative error between them approaches to $15 \%$ in the stable interval for Seebeck coefficient when the thermoelectric module is independently controlled by the gate voltage. This work can provide an additional way for solving the precise control of the performance for thermoelectric devices.
\end{abstract}

Index Terms-Double-voltage-control mechanism, effective thermal conductivity model, graphene, thermoelectric cooling.

\section{INTRODUCTION}

$\mathrm{T}_{1}$ HE demands for higher data rate of SoC (System on chip) led to a continuously increasing power consumption and thus more heat dissipation on chips [1-3]. The resultant high

This work was supported by NSFC (61331004), Director Fund of Key Laboratory of Ministry of Education of Design and Electromagnetic Compatibility of High Speed Electronic System under Grant 2014001. The authors are with the Shanghai Key Lab of Modern Optical System, University of Shanghai for Science and Technology, Shanghai, China, 200093. (E-mail: nwang@usst.edu.cn).

Ning Wang, Hong-Wen Li, Hong-Zhi Jia, Zhong-Dao Ren and Zi-Yi Zhao are with Engineering Research Center of Optical Instrument and System, Ministry of Education, Shanghai Key Lab of Modern Optical System, University of Shanghai for Science and Technology, Shanghai, China (hzjia@usst.edu.cn)

Can Ding is with the Global Big Data Technologies Centre (GBDTC), University of Technology Sydney (UTS), Sydney, NSW, Australia, 2007 (Email: can.ding.1989@gmail.com).

Li-Yun Shi is with Key Laboratory of Ministry of Education of Design and EMC of High-Speed Electronic Systems, Shanghai Jiao Tong University, Shanghai, China (2683678780@qq.com) temperature can sometimes significantly deteriorate the system performance. Especially the so-called local hot spots produced by the non-uniform power density distribution on chips can easily destroy the electron component structure due to the high temperature. A statistic report in [4] demonstrates that more than $55 \%$ of the electronic equipment failure is attributed to this over-heat problem. As the surface temperature increasing, the component failure rate is dramatically increased, which is a significant threaten to the entire system. Therefore, solving this thermal issue is a life-saving task for SoC.

Thermoelectric cooling is a new type of refrigeration technology advocated for its small size, light weight, no noise, long service life, and easiness of controlling and integration [5]. Thus it has a potential to solve thermal issues for high power chips and devices.

In a long time, it was a basic requirement of having a precisely controlled thermal transfer of thermoelectric materials in the thermal managements for on-chip and power devices [6], [7]. However, thermoelectric materials usually have fixed thermal conductivity properties. Thermoelectric materials with naturally variable thermal conductivity properties are rare. Many works were done to reduce the thermal conductivity of thermoelectric materials by introducing phonon scattering mechanism [8], [9]. The reduced phonon thermal conductivity was generally attributed to the formation of point defects [10] and nanostructuring with the lattice interface [11-14]. The way of point defects usually employs the methods of alloy and doping, and the latter can not only introduce the short-range disordered semiconductor but also can improve the electrical performance due to high carrier concentration [15]. In 1993, Hicks and Dresselhaus [16] demonstrated a fact that the characters of some materials can be changed by preparing them in the form of multilayered superlattices, since the electrons are confined to move in two dimensions and the layering reduces the phonon thermal conductivity by scattering between layers. Then, Koga et al. found that the thermal conductivities of GaAs/AlAs superlattices are much smaller than those of bulk materials, and they can be further reduced with a thinner well layer and barrier layer [17]. It is the superlattice structure which improves the thermal conductivity of the material due to the quantum confinement effect of the phonon. Available studies mainly adjusted the conductivity through superlattice structures and phonon scattering from process method in internal 
thermoelectric material. However, once thermoelectric materials or devices are fabricated, the conductivity cannot be regulated anymore in a normal work process.

Meanwhile, graphene was used as a new type potential thermoelectric material [18-20] since it has an unique electronic band structure, carrier density, and polarity with electronically tunable features. Both theoretical studies [21-23] and experiments [24-26] demonstrate that the seebeck coefficient of graphene is quite large, which can reach up to $\pm 100 \mu \mathrm{VK}^{-1}$. Meanwhile, the seebeck coefficient of graphene can be controlled by the gate voltage. A suspended graphene with a large conductivity of $3000 \mathrm{~W} / \mathrm{mK}$ and a large thermal conductivity of $5000 \mathrm{~W} / \mathrm{mK}$ was presented in [27]. The excellent thermal conductivity results in bad thermoelectric figure of merit. There are many works focusing on reducing the thermal conductivity of graphene as much as possible and they are used for thermoelectric energy conversion [28]. Bae et al. observed a fact that the heat transportation in the device is dominated by the thermal resistance of $\mathrm{SiO}_{2}$ due to the large size difference, and in reality, the thermal conductance should be replaced by an overall thermal conductance per unit length, which is about $18 \mathrm{~W} / \mathrm{mK}$ [29]. In [30], Cao et al. comprehensively investigated the influence of the thermal resistance at the nanoscale constriction on thermal transport using non-equilibrium molecular dynamics method, and for the first time reported the fact that the characteristics of the thermal resistance arising from single-nanoconstriction is inversely proportional to the constriction width [30]. This indicates that graphene is a promising thermoelectric material to solve the over-heat problem in SoC.

In this paper, an effective thermal conductivity model of graphene is proposed with a double-voltage-controlled mechanism for thermoelectric cooling. The combination of gate voltage and externally applied voltage can provide an additional means to manipulate the thermoelectric properties of graphene.

\section{MATHEMATIC MODEL}

\section{A. Theoretical Basis}

Fig. 1 gives a schematic model of the proposed graphene thermoelectric cooling element. Thermoelectric phenomena generally involves three processes: Seebeck, Peltier, and Thomson effects. Seebeck effect is the generation of a voltage due to a temperature difference between two different conductors or semiconductors. It is quantified by the Seebeck coefficient, $S=-\Delta V / \Delta T$, to represent the ability of power generating. Peltier effect is regarded as an inverse process of Seebeck effect, where both cooling and heating can be excited when the electric current flows through the same heterogeneous material surface. The relationship between Seebeck coefficient and Peltier coefficient can be described by Thomson effect, that is $\Pi=S T_{r}$, where $T_{r}$ is the reference temperature. The above mentioned three effects form into a basic working mechanism of the proposed thermoelectric device.
According to the first law of thermodynamics, the temperature distribution on the surface of thermoelectric materials can be determined by

$$
C_{p} \frac{d T}{d t}=\frac{d}{d x}\left(\kappa \frac{d T}{d x}\right)-\left(T_{a} \frac{d S}{d T}\right) J_{e} \frac{d T}{d x}+\frac{J_{e}^{2}}{\sigma},
$$

where $C_{P}, T_{a}, K, S, J_{e}$ and $\sigma$ are the specific heat per unit volume at constant pressure, absolute temperature, thermal conductivity, Seebeck coefficient, coupled charge current flux, and electrical conductivity, respectively. Compared to the ordinary heat conduction equation, a mathematical term $\left[T_{a}(d S / d T)\right] J_{e}(d T / d x)$ was added in (1). This term comes from Thomson effect suggesting that distributed heating or cooling can occur even in the same solid due to the temperature dependence of Seebeck coefficient. However, this study is focused on low-temperature system within the thermal conduction for thermoelectric cooling, the influence of Thomson effect can be neglected [31]. In addition, impurity scattering, defect's scattering, and phonon-phonon scattering are the other dominate factors affecting thermal conductivity at the low temperatures [32-36]. The contribution of phonons to thermal conductivity varies as the temperature changing when the temperature is high [36], [37]. This work only considers room temperature situations with a small variation, the effects of the scattering mechanism and the contribution of the phonon on the thermal conductivity are neglected and the thermal conductivity is nearly constant.

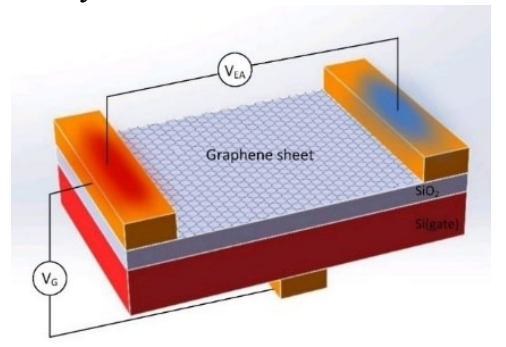

Fig. 1. Schematic of graphene thermoelectric cooling element. Graphene sheet (length and width $l_{g r}=7 \mu \mathrm{m}, s_{g r}=4 \mu \mathrm{m}$ ) is deposited on $\mathrm{SiO}_{2}$ (thickness $t_{o x}=300$ $\mathrm{nm}$ ) with a highly doped Si substrate as the back-gate.

With an external voltage $V_{E A}$ applied to the thermoelectric device as shown in Fig. 1, the energy with heat conduction will be generated at the two sides of element as Peltier effect, which is equal to $S T I$. At the same time, the cooling effect is opposed by the heat conduction at the rate of $k_{g r} r_{g r} \Delta T / l_{g r}$. Considering the state of the joule heat, half of the Joule heat $\left(I^{2} R_{g r} / 2\right)$ passes to the sink and the rest to the source. The heat transport in both sides of the thermoelectric cooling unit are as follows:

$$
\begin{aligned}
& Q_{c}=S T_{c} I-\frac{k_{g r} s_{g r}}{l_{g r}} \Delta T-\frac{1}{2} I^{2} R_{g r} \\
& Q_{h}=S T_{h} I-\frac{k_{g r} s_{g r}}{l_{g r}} \Delta T+\frac{1}{2} I^{2} R_{g r}
\end{aligned}
$$

where $Q_{c}, Q_{h}, k_{g r}, s_{g r}, l_{g r}, T_{c}, T_{h}, R_{g r}$ and $I$ are heat transfers at cool side and hot side, thermal conductivity, material cross-sectional area, length of the material, temperature at both sides, resistance, and electrical current, respectively. $\Delta T$ is the temperature difference between the sides of 1 and 2 , represented by $\Delta T=T_{h}-T_{c}$. 
For a traditional thermoelectric cooling element, it can be seen as one thermoelectric material to study the overall thermal behavior. According to the Fourier's law of conduction, the equivalent thermal conductivity for the entire thermoelectric cooling system is given by

$$
k_{\text {eff }}=\frac{Q_{T E C l} l_{g r}}{s_{g r} \Delta T} \text {. }
$$

However, the heat transfer is not equal on both the two sides of TEC. The equivalent heat transfer through the thermoelectric unit $Q_{T E C}$ is calculated by

$$
Q_{T E C}=\frac{Q_{h}+Q_{c}}{2} \text {. }
$$

It is known that the current that flows through the thermoelectric unit can be expressed by the external applied voltage $V_{E A}$ as

$$
I=\frac{V_{E A}-S \Delta T}{R_{g r}}
$$

Therefore, based on equations (2) to (7), the effective thermal conductivity $k_{e f f}$ can be obtained as,

$$
k_{e f f}=\frac{S V_{E A} T l_{g r}}{s_{g r} R_{g r} \Delta T}-\frac{S^{2} T l_{g r}}{s_{g r} R r}-k_{g r}
$$

where $T$ is average temperature $T=\left(T_{h}+T_{c}\right) / 2$. From the Eq. (8), it is concluded that when the thermoelectric material characters including size and thermoelectric parameters are determined, the effective thermal conductivity of the thermoelectric module can be controlled by the external applied voltage $V_{E A}$.

\section{B. Seebeck Coefficient and Square Resistance of Graphene}

Due to the influence of gate voltage, the Seebeck coefficient and the square resistance of graphene both exhibit modulation characteristics [38]. Based on the properties, the graphene thermoelectric cooling element shown in Fig. 1 was studied in this paper. Based on this module, the influence of the unique properties of graphene on thermoelectric module was investigated.

Following the definitions of charge density and Fermi level, the closed-form approximation of the Seebeck coefficient in graphene was derived from the semi-classical Mott relationship considering the effect of the gate voltage for carriers, consistent with the model proposed by Dorgan [39].

$$
S=-\frac{2 \pi^{\frac{3}{2}} k_{B}^{2} T}{3 q \hbar v_{F}} \frac{\frac{C_{O X} V_{G D}}{q} \sqrt{\left|\frac{C_{O X} V_{G D} \mid}{q}\right|}}{\left(\frac{C_{O X} V_{G D}}{q}\right)^{2}+4 n_{0}^{2}}
$$

where $T, k_{B}, v_{F}, \hbar, q$, and $n_{0}$ are the reference temperature, Boltzmann constant, Fermi velocity, reduced Planck's constant, electron charge, and carrier density, respectively. According to [38], the carrier density $n_{0}$ is equal to $1.8 \times 10^{11} \mathrm{~cm}^{-2}$. And $C_{o x}=\varepsilon_{0} \varepsilon_{r} / t_{o x}$ is the capacitance per unit area (quantum capacitance can be neglected here [40], [41]), $\varepsilon_{0}$ and $\varepsilon_{r}$ are the vacuum dielectric constant and relative dielectric constant, respectively. $t_{o x}$ is the thickness of the base. $V_{G D}=V_{G^{-}} V_{D}$, where $V_{G}$ is the gate voltage and $V_{D}$ is the neutral (Dirac) voltage. Fig.
2 shows the regulation relationship between the Seebeck coefficient and gate voltage with different temperatures. At the point where $V_{G D}=0$ and the Seebeck coefficient is about 0 , the characteristic of material is more like a conductor. On the contrary, with the absolute value of the gate voltage increasing, the Seebeck coefficient tends to be stable, presenting a thermoelectric semiconductor suit to cooling study.

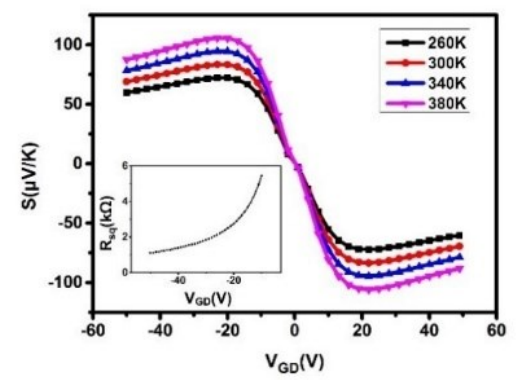

Fig. 2. Seebeck coefficient of graphene as a function of $V_{G D}$ from (9) with different reference temperatures. The inset shows the impact about $V_{G D}$ on the square resistance of graphene from (10).

Meanwhile, considering the effect of the gate voltage for carriers, the square resistance is denoted as simple form $R_{s q}$, which is consistent with the model proposed by Grosse [38]

$$
R_{s q}=\frac{1}{-q \mu \sqrt{\left(\frac{C_{O X} V_{G D}}{q}\right)^{2}+4 n_{0}{ }^{2}}},
$$

where $\mu=3230 \mathrm{~cm}^{2} / \mathrm{V} \cdot \mathrm{S}$ is the mobility [38]. Hence, the Seebeck coefficient and square resistance of graphene can be both controlled by the gate voltage. It is then deduced that the effective thermal conductivity of its two-dimensional plane can also be controlled by gate voltage. Considering the relationship between effective thermal conductivity and external applied voltage, a double-voltage-controlled effective thermal conductivity model can be obtained. Substituting (9) and (10) into (8), the function of this model is described as follows:

$$
\begin{gathered}
k_{e f f}= \\
\frac{2 \pi^{\frac{3}{2}} k_{B}^{2} T^{2} \mu V_{E A}}{3 t h_{g r} \hbar v_{F} \Delta T} \frac{\frac{C_{O X} V_{G D}}{q} \sqrt{\left|\frac{C_{O X} V_{G D}}{q}\right|}}{\sqrt{\left(\frac{C_{O X} V_{G D}}{q}\right)^{2}+4 n_{0}^{2}}}+\frac{4 \pi^{3} k_{B}^{4} T^{3} \mu}{9 t h_{g r} \hbar^{2} v_{F}^{2}} \frac{\left(\frac{C_{O X} V_{G D}}{q}\right)^{2}\left|\frac{C_{O X} V_{G D} \mid}{q}\right|}{\left[\left(\frac{C_{O X} V_{G D}}{q}\right)^{2}+4 n_{0}{ }^{2}\right]^{\frac{3}{2}}}- \\
k_{g r}
\end{gathered}
$$

where $t h_{g r}$ is the thickness of graphene. Modulating the gate voltage added to the graphene is to tune the Fermi energy and change the hybridization. As a consequence, the Seebeck coefficient and square resistance are further modulated on the external features. And modulating external applied voltage is to change the working condition of the thermoelectric cooling device. Both behaviors will alter the equivalent heat transfer through the thermoelectric unit $Q_{T E C}$ and temperature difference $\Delta T$. According to (11), it is conducted that the effective thermal conductivity of the whole cooling unit is controlled together by the gate voltage and the external applied voltage when the material is determined. 


\section{EleCtronic SimUlation HSPICE Model}

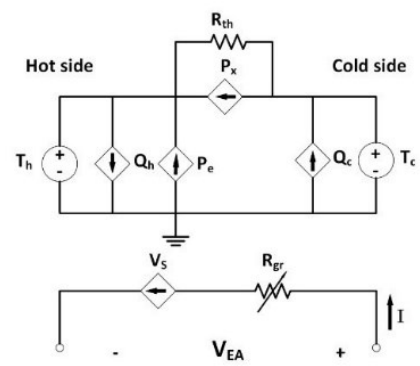

Fig. 3. Three-port circuit model for a TEC element. (In the process of HSPICE modeling, the devices that are related with the Seebeck coefficient and square resistance of graphene are all regarded as controlled sources with voltage. Hence, many components in the simulation circuits are all controlled by the two independent voltage sources.).

TABLE I

Analogies between Thermal Variable and Electrical Variable

Thermal variable Electrical variable
Heat flow $Q(W)$

Temperature $\mathbf{T}(\mathbf{K})$

Thermal resistance $R_{t h}(K / W)$
Current flow I(A)

Voltage V(V)

Electrical resistance $\mathbf{R}(\boldsymbol{\Omega})$
To verify the accuracy of the proposed model, numerical simulations on HSPICE electrical model were conducted. Based on the thermal and electrical mapping relationship which can be referred to TABLE I [42], [43], a circuit model is presented in Fig. 3. In the figure, $T_{c}, T_{h}, Q_{h}, Q_{c}$, and $R_{t h}$ represent cold junction voltage, hot junction voltage, hot junction controlled current sources, cold junction controlled current sources, and thermal resistance of the graphene thermoelectric cooling, respectively. $P_{e}$ and $P_{x}$ are the equivalent electrical input powers.

$$
\begin{aligned}
& P_{e}=Q_{h}-Q_{c}=I^{2} R_{g r}+I S\left(T_{h}-T_{c}\right) \\
& P_{x}=S I T_{c}-\frac{1}{2} I^{2} R_{g r}
\end{aligned}
$$

The electrical behavior in the model is caused by the Seebeck and Joule effects, so the external applied voltage can be described as

$$
V_{E A}=I R_{g r}+V_{S}=I R_{g r}+S\left(T_{h}-T_{c}\right) .
$$

\section{RESULTS AND DISCUSSION}

In order to study the thermoelectric behavior of the thermoelectric cooling unit, the maximum temperature difference mode $\left(T_{h}=300 \mathrm{~K}, Q_{c}=0 \mathrm{~W}\right)$ was conducted with the same operation in [43], [44]. In this mode, the effect of the applied voltage and gate voltage on the effective thermal conductivity for the whole cooling unit is obtained. In this work, the lateral air radiation and heat conduction can be neglected due to the small environment parameter variations in the process of model construction.

In Fig. 4 (a), it can be seen that the temperature difference between the hot and cold junctions becomes larger with the increase of the external input voltage while the gate voltage is fixed, which means the Seebeck coefficient and square resistance in graphene are fixed as well. Opposite to the growth rate of the temperature difference, the effective thermal conductivity $k_{\text {eff }}$ of the module becomes larger and larger with the increase of the external input voltage. With the small change of $V_{E A}$, the number of $k_{\text {eff }}$ can span from 0 to 1000 $\mathrm{W} / \mathrm{mK}$. That is to say, the dependency effect of $k_{\text {eff }}$ on $V_{E A}$ is significant, which can be denoted as a controlling mechanism. However, $\Delta T$ is not the same case since the number only varies from 0 to 0.78 due to the small excited voltage.

For the gate voltage applied to graphene, the regulation degree on $k_{\text {eff }}$ by $V_{G D}$ is not obvious with large negative voltage. As shown in Fig. 4 (b), small variations of 650 (from 1600 to $950 \mathrm{~W} / \mathrm{mK}$ ) was observed when $V_{G D}$ increase from $-50 \mathrm{~V}$ to $-20 \mathrm{~V}$ with the a fixed $V_{E A}$. Moreover, the decrease of $\mathrm{k}_{\text {eff }}$ has a limitation when $V_{G D}$ reaches $-20 \mathrm{~V}$, then it rises dramatically with $V_{G D}$ continuously increasing to $-10 \mathrm{~V}$.

Fig. 5 shows the impact of the external applied voltage $V_{E A}$ and the gate voltage $V_{G D}$ on the effective thermal conductivity $k_{\text {eff }}$ of the thermoelectric module synchronously. Based on the proposed double-voltage-control model, both coarse-tuning and fine-tuning of effective thermal conductivity can be realized.
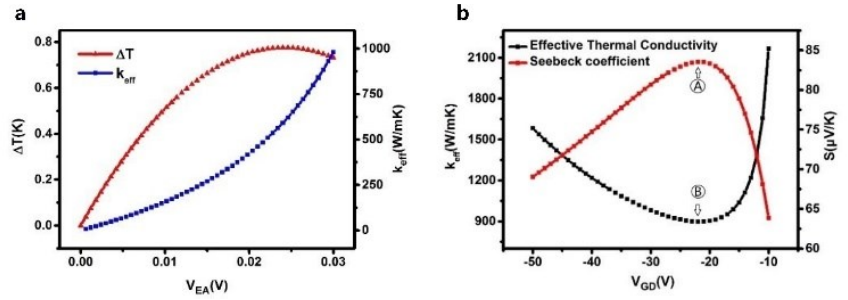

Fig. 4. (a) Voltage-controlled relationships for the temperature difference and effective thermal conductivity when $\mathrm{V}_{\mathrm{GD}}=-30 \mathrm{~V}$. (b) Gate-voltage-controlled effective thermal conductivity and Seebeck coefficient in graphene based thermoelectric devices when $\mathrm{V}_{\mathrm{EA}}=0.03 \mathrm{~V}$. Point $\mathrm{A}(\max \mathrm{S})$ and point $\mathrm{B}(\min$ keff) is a couple data in calculations.

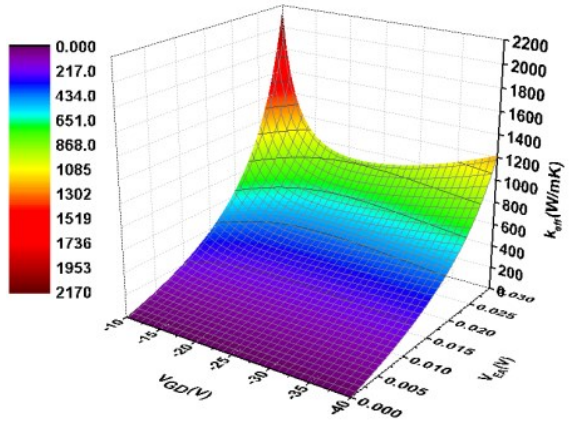

Fig. 5. 3-D data model of the effective thermal conductivity $k_{\text {eff }}$ controlled by double-voltage.
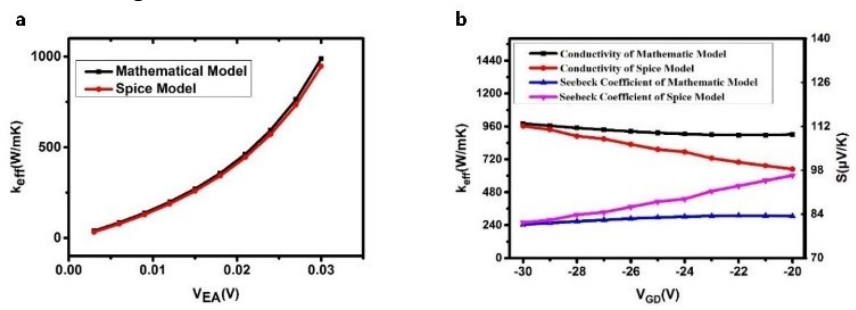

Fig. 6. (a) Comparison results about the effective thermal conductivity of mathematical model and circuit simulation model using HSPICE under the $\mathrm{V}_{\mathrm{EA}}$ controlling alone $\left(\mathrm{V}_{\mathrm{GD}}=-30 \mathrm{~V}\right)$. (b) Model Comparisons about the effective thermal conductivity and Seebeck coefficient with mathematical model and SPICE model under the $\mathrm{V}_{\mathrm{GD}}$ controlling alone $\left(\mathrm{V}_{\mathrm{EA}}=0.03 \mathrm{~V}\right)$. 
First, large scale control of $k_{\text {eff }}$ can be carried out in coarse-tuning mode by changing $V_{E A}$. Once $V_{E A}$ is determined, the tuning scale is fixed. In this mode, fine tuning of $k_{\text {eff }}$ can be realized by spanning $V_{G D}$ from -40 to $-22 \mathrm{~V}$, and a large scale tuning of $k_{\text {eff }}$ can be achieved with $V_{G D}$ spanning from -22 to - 10 V. Meanwhile, we can see that it is only double-voltages keep the extreme values synchronously with the two small or large voltages that optimized $k_{\text {eff }}$ can be obtained from Fig. 5 .

As shown in Fig. 6(a), when $k_{\text {eff }}$ is individually modulated by $V_{E A}$, the proposed $k_{\text {eff }}$ model has a good agreement with the HSPICE simulation results. The maximum discrepancy between the two methods is only $4 \%$.

However, as shown in Fig. 6 (b), when $k_{\text {eff }}$ is individually modulated by $V_{G D}$, the discrepancy between the proposed model and HSPICE model gets larger as $V_{G D}$ increases from -30 to $-20 \mathrm{~V}$. Note that it is only the Seebeck coefficient remains stable around $-25 \mathrm{~V}$ for $V_{G D}$ that the graphene can be regarded as thermoelectric semiconductor rather than the intrinsic semiconductor. So the characteristic of thermoelectric in graphene will become more significant when large negative voltage of gate source is applied. The relative error between the two models is $15 \%$ with $V_{G D}$ of $-25 \mathrm{~V}$, which is larger than the one of $V_{E A}$ regulation, still within a certain consistency. With the increase of absolute value of $V_{G D}$, the agreement between two models fits well due to the stable Seebeck coefficient range. From the above two different voltage regulation mechanism and the HSPICE simulation results, the feasibility of the proposed double-voltage-controlled effective thermal conductivity model is demonstrated.

As shown in Fig. 7 (a), the effect of $V_{G D}$ on the figure of the merit ZT for graphene on $\mathrm{SiO}_{2}$ is given, where the larger Seebeck coefficient can be obtained. When $V_{G D}$ is close to -10 $\mathrm{V}, \mathrm{ZT}$ decreases dramatically. Thus, it is necessary to improve the absolute voltage for $V_{G D}$ to enhance the thermoelectric performance. Meanwhile, ZT also goes up with the temperature rising. Besides, since the size comparison between the graphene and the substrate is so obvious that the heat transport is affected by the Si substrate due to its good heat dissipating path. Hence, given the geometry of the device, graphene is considered as a one-dimensional diffusive heat transport channel, where both the junctions are treated as point sources for dissipating and $\mathrm{SiO}_{2}$ acts as a path for heat flow from the graphene into the $\mathrm{Si}$ thermal bath. Thus the thermal conductance should be replaced by an overall thermal conductance per unit length, $k_{o}=1 /\left[\mathrm{L}_{\mathrm{HL}}\left(\mathrm{R}_{\mathrm{OX}}+\mathrm{R}_{\mathrm{Si}}\right) \approx 18 \mathrm{~W} / \mathrm{mK}\right.$, where $L_{H L}=\left(\mathrm{t}_{\mathrm{ox}} \mathrm{th}_{\mathrm{gr}} \mathrm{k} / \mathrm{k}_{\mathrm{ox}}\right)^{1 / 2} \approx 0.2 \mu \mathrm{m}$ is healing length which is a measure of the temperature diffusion from a heat source along the graphene [29], [45]. The impact of the external applied voltage $V_{E A}$ and the gate voltage $V_{G D}$ on the new effective thermal conductivity $k_{e s}$ of the thermoelectric module synchronously is shown in Fig. 7 (b).

Comparing Fig. 5 with Fig. 7 (b), it is observed that the effective thermal conductivity is reduced noticeably from 897 $\mathrm{W} / \mathrm{mK}$ for $k_{\text {eff }}$ to $29 \mathrm{~W} / \mathrm{mK}$ for $k_{\text {es }}$ when $V_{G D}=-21 \mathrm{~V}$ and $V_{E A}=0.03 \mathrm{~V}$. But the variation trend is almost the same after the adjustment considering the influence of substrate effect.
Therefore, the effective thermal conductivity considering the substrate dissipation path is smaller than the one with graphene sheet. This effective thermal conductivity is more suitable for the double-voltage-controlled mechanism.
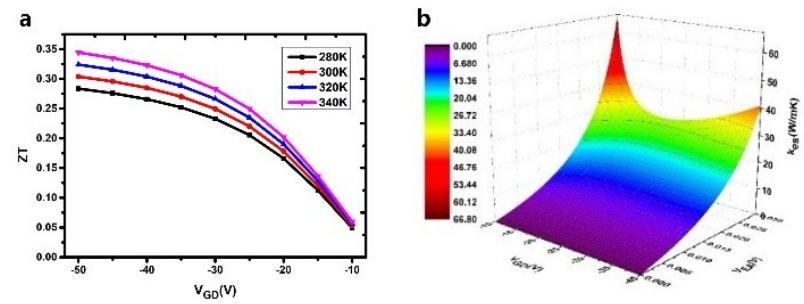

Fig. 7. (a) The figure of the merit (ZT) for graphene on $\mathrm{SiO}_{2}$ as a function of $V_{G D}$ in the different reference temperatures. (b) 3-D data model of the effective thermal conductivity $k_{e s}$ controlled by double-voltage. (Considering the influence of $\mathrm{SiO}_{2}$ and $\mathrm{Si}$ thermal bath when analyzing the heat transport.)

\section{CONCLUSIONS}

Based on the combination of the unique properties of graphene material and the effective thermal conductivity model of the thermoelectric cooling unit controlled by the external applied voltage, a thermoelectric model of effective thermal conductivity of graphene is proposed with both the external applied voltage and gate voltage. The thermal behavior control based on the external applied voltage and the gate is discussed. The correctness of the theoretical model is verified by HSPICE circuit simulation model. The discrepancy between the two models is only $4 \%$ with independent adjustment of $V_{E A}$, and the other one reaches $15 \%$ with $V_{G D}$ of $-25 \mathrm{~V}$ at stable Seebeck coefficient point. The proposed effective thermal conductivity model with double-voltage-controlled property mechanism can provide a new way for graphene based thermoelectric cooling technology.

\section{REFERENCES}

[1] L. Jing, Y. H. Cheng and J. F. M, "Analysis and Optimization of Thermal-Driven Global Interconnects in Nanometer Design," IEEE Transactions on Components, Packaging and Manufacturing Technology, vol. 1, no. 10, pp. 1564-1572, Oct. 2011.

[2] W. H. Park and C. K. Yang, "Effects of Using Advanced Cooling Systems on the Overall Power Consumption of Processors," IEEE Transactions on Very Large Scale Integration (VLSI) Systems, vol. 21, no. 9, pp. 1644-1654, Sept. 2012.

[3] A. Samake, P. Kocanda and A. Kos, "A new idea of effective cooling of integrated circuits," in 2016 International Conference on. Signals and Electronic Systems, Krakow, Poland, pp.179-182.

[4] L. T. Yeh, "Review of Heat Transfer Technologies in Electronic Equipment," Journal of Electronic Packaging, vol. 117, no. 4, pp. 333-339, Dec. 1995.

[5] G. E. Bulman, P. M. Thomas, G. Krueger, R. Venkatasubramanian, D. A. Marckx and B. Ratliff, "High Heat Flux, High Temperature Cooling of Electronics With Thermoelectric Devices," IEEE Electron Device Letters, vol. 35, no. 4, pp. 476-478, Apr. 2014.

[6] S. C. Lin and K. Banerjee. "Cool Chips: Opportunities and Implications for Power and Thermal Management," IEEE Transactions on Electron Devices, vol. 55, no. 1, pp. 245-255, Jan. 2008.

[7] K. J. Chen; C. H. Chao and A. A. Wu, "Thermal-Aware 3D Network-On-Chip (3D NoC) Designs: Routing Algorithms and Thermal Managements," IEEE Circuits and Systems Magazine, vol. 15, no. 4, pp. 45-69, Fourthquarter. 2015. 
[8] T. C. Harman, P. J. Taylor, M. P. Walsh and B. E. Laforge, "Quantum dot superlattice thermoelectric materials and devices," Science, vol. 297, no. 5590, pp. 2229-2232, Sept. 2002.

[9] S. Lee, K. Esfarjani, J. Mendoza, M. S. Dresselhaus and G. Chen, "Lattice thermal conductivity of $\mathrm{Bi}, \mathrm{Sb}$, and $\mathrm{Bi}-\mathrm{Sb}$ alloy from first principles," Physical Review B, vol. 89, no. 8, pp. 188-192, Feb. 2014.

[10] G. J. Snyder, E. S. Toberer, "Complex thermoelectric materials," in Nanoscience and technology: A Collection of Reviews from Nature Journals, 2008, pp. 50-59.

[11] A. Pattamatta and C. K. Madnia, "Modeling heat transfer in $\mathrm{Bi}_{2} \mathrm{Te}_{3}-\mathrm{Sb}_{2} \mathrm{Te}_{3}$ nanostructures," International Journal of Heat and Mass Transfer, vol. 52, no. 3-4, pp. 860-869. Jan. 2009.

[12] H. Li, Y. Yu and G. Li, "Computational modeling and analysis of thermoelectric properties of nanoporous silicon," Journal of Applied Physics, vol. 115, no.12, pp. 42-47, Mar. 2014.

[13] S. Bathula, B. Gahtori, M. Jayasimhadri and S. K. Tripathy, "Microstructure and mechanical properties of thermoelectric nanostructured n-type silicon- germanium alloys synthesized employing spark plasma sintering," Applied Physics Letters, vol. 105, no. 6, pp. 0619021-0619025, Aug. 2014.

[14] Y. Lan, A. J. Minnich, G. Chen and Z. Ren, "Enhancement of Thermoelectric Figure-of-Merit by a Bulk Nanostructuring Approach," Advanced Functional Materials, vol. 20, no. 3, pp. 357-376, Dec. 2009.

[15] A. J. Minnich, M. S. Dresselhaus, Z. F. Ren and G. Chen, "Bulk nanostructured thermoelectric materials: current research and future prospects," Energy and Environmental Science, vol. 2, no. 5, pp. 466-479, May. 2009.

[16] L. D. Hicks and M. S. Dresselhaus, "Effect of quantum-well structures on the thermoelectric figure of merit," Physical Review B Condensed Matter, vol. 47, no. 19, pp. 12727-12731, May. 1993.

[17] T. Koga, X. Sun, S. B. Cronin and M. S. Dresselhaus, "Carrier Pocket Engineering to Design Superior Thermoelectric Materials Using GaAs/AlAs Superlattices," Applied Physics Letters, vol. 73, no. 20, pp. 2950-2952. Nov. 1998.

[18] K. S. Novoselov, A. K. Geim, S. V. Morozov, D. Jiang, Y. Zhang, S. V. Dubonos, I. V. Grigorieva and A. A. Firsov, "Electric Field Effect in Atomically Thin Carbon Films," Science, vol. 306, no. 5696, pp. 666-669, Oct. 2004.

[19] A. H. Castro Neto, F. Guinea, N. M. R. Peres, K. S. Novoselov and A. K. Geim, "The electronic properties of graphene," Reviews of Modern Physics, vol. 81, no. 1, pp. 109-162, Jan. 2009.

[20] S. Das Sarma, Shaffique Adam, E. H. Hwang, and Enrico Rossi, "Electronic transport in two-dimensional graphene," Reviews of Modern Physics, vol. 83, no. 2, pp. 407-476, May. 2011.

[21] Y. Ouyang and J. Guo, "A theoretical study on thermoelectric properties of graphene nanoribbons," Applied Physics Letters, vol. 94, no. 26, pp. 2631071-2631073, Jun. 2009.

[22] X. Z. Yan, Y. Romiah and C. S. Ting, "Thermoelectric power of Dirac fermions in graphene," Physical Review B, vol. 80, no.16, pp. 1654321-1654326, Oct. 2009.

[23] E. H. Hwang, E. Rossi and S. Das Sarma, "Theory of thermopower in two-dimensional graphene," Physical Review B, vol. 80, no. 23, pp. 2354151-2354156, Dec. 2009.

[24] Y. M. Zuev, W. Chang and P. Kim, "Thermoelectric and Magnetothermoelectric Transport Measurements of Graphene," Physical Review Letters, vol. 102, no. 9, pp. 0968071-0968074, Mar. 2009.

[25] P. Wei, W. Z. Bao, Y. Pu, C. N. Lau and J. Shi, "Anomalous thermoelectric transport of dirac particles in graphene," Physical Review Letters, vol. 102, no. 16, pp. 16680801-16680816, Apr. 2009.

[26] J. G. Checkelsky and N. P. Ong, "Thermopower and Nernst effect in graphene in a magnetic field," Physical Review B, vol. 80, no. 8, pp. 0814131-0814134, Aug. 2009.

[27] J. H. Seol, I. Jo, A. L. Moore, L. Lindsay, Z. H. Aitken, M. T. Pettes, X. Li, Z. Yao, R. Huang, D. Broido, N. Mingo, R. S. Ruoff and L. Shi. "Two-Dimensional Phonon Transport in Supported Graphene," Science, vol. 328, no. 9, pp. 213-216, Nov. 2010.

[28] A. A. Balandin, "Thermal properties of graphene and nanostructured carbon materials," Nature Materials, vol. 10, no. 8, pp. 596-581, Jul. 2011.

[29] M. H. Bae, Z. Y. Ong, D. Estrada and E. Pop, "Imaging, Simulation, and Electrostatic Control of Power Dissipation in Graphene Devices" Nano Letters, vol. 10, no. 12, pp. 4787-4793, Jun. 2010.

[30] B. Y. Cao, W. J. Yao, Z. Q. Ye, "Networked nanoconstrictions: An effective route to tuning the thermal transport properties of graphene," Carbon, vol. 96, pp. 711-719, Jan. 2016.
[31] E. Massaguer, A. Massaguer, L. Montoro and J. R. Gonzalez, "Development and validation of a new TRNSYS type for the simulation of thermoelectric generators," Applied Energy, vol. 134, no. 6, pp. 65-74, Dec. 2014.

[32] J. H. Chen, C. Jang, M. S. Fuhrer, E. D. Williams and M. Ishigami, "Charged-impurity scattering in graphene," Nature Physics, vol. 4, no. 5, pp. 377-381, Apr. 2008.

[33] J. H. Chen, W. G. Cullen, C. Jang, M. S. Fuhrer and E. D. Williams, "Defect Scattering in Graphene," Physical Review Letters, vol. 102, no. 23, pp. 2368051-2368052. Jun. 2009.

[34] M Morsin and Y Yusof, "The effect of defect on thermal conductance of single layer graphene transistor," in SCOReD, Batu Ferringhi, Malaysia, 2014, pp. 1-4.

[35] P. Dollfus, V. H. Nguyen, V. T. Tran, M. C. Nguyen, A. Bournel and J. S. Martin, "Thermoelectric effects in graphene and graphene-based nanostructures using atomistic simulation," in PATMOS, Bremen, Germany, 2016, pp. 38-43.

[36] F. Mazzamuto, J. Saint-Martin, V. Hung Nguyen, Y. Apertet, C. Chassat, and P. Dollfus, "Nanostructuration of Graphene Nanoribbons for thermoelectric applications," SISPAD, Osaka, Japan, 2011, pp. 223-226.

[37] G. S. Kliros and P. C. Divari, "Thermoelectric performance of electrons in ballistic graphene ribbons," in 2011 International Semiconductor Conference, Sinaia, Romania, pp. 45-48.

[38] K. L. Grosse, M. H. Bae, F. Lian, E. Pop and W. P. King, "Nanoscale Joule heating, Peltier cooling and current crowding at graphene-metal contacts," Nature Nanotechnology, vol. 6, no. 5, pp. 287-290, Apr. 2011.

[39] V. E. Dorgan, M. H. Bae and E. Pop, "Mobilityand saturation velocityin graphene on $\mathrm{SiO}_{2}$," Applied Physics Letters, vol. 97, no. 8, pp. 0821121-0821123, Aug. 2010.

[40] T. Fang, A. Konar, H. Xing and D. Jena, "Carrier statistics and quantum capacitance of graphene sheets and ribbons," Applied Physics Letters, vol. 91, no. 9, pp. 0921091-0921093. Aug. 2007.

[41] W. J. Zhu, V. Perebeinos, M. Freitag, and P. Avouris, "Carrier scattering, mobilities, and electrostatic potential in monolayer, bilayer, and trilayer graphene," Physical Review B, vol. 80, no. 23, pp. 23540201-23540218, Dec. 2009.

[42] J. A. Chavez, J. A. Ortega, J. Salazar, A. Turó and M. J. Garcia, "SPICE model of thermoelectric elements including thermal effects," IEEE Instrumentation and Measurement Technology Conference, vol. 2, no. 2, pp. 1019-1023, Feb. 2000.

[43] D. Mitrani, J. Salazar, A Turó, M. J. García and J. A. Chávez, "One-dimensional modeling of TE devices considering temperature-dependent parameters using SPICE," Microelectronics Journal, vol. 40, no. 9, pp. 1398-1405, Sept. 2009.

[44] S. Lineykin and S. Ben-Yaakov, "Analysis of thermoelectric coolers by a spice-compatible equivalent-circuit model," IEEE Power Electronics Letters, vol. 3, no. 2, pp. 63-66, Jun. 2005.

[45] I. J. Vera-Marun, J. J. van den Berg, F. K. Dejene and B. J. van Wees, "Direct electronic measurement of Peltier cooling and heating in graphene," Nature Communications, vol. 7, no. 11525, pp. 1-6, May. 2016. 